\title{
AN ACYCLIC ANALOGUE TO HEAWOOD'S THEOREM
}

\author{
by MICHAEL O. ALBERTSON* and DAVID M. BERMAN
}

(Received 25 April, 1977; revised 12 October, 1977)

1. Introduction. The concept of acyclic coloring was introduced by Grünbaum [5] and is a generalization of point arboricity.

A proper $k$-coloring of the vertices of a graph $G$ is said to be acyclic if $G$ contains no two-colored cycle. The acyclic chromatic number of a graph $G$, denoted by $a(G)$, is the minimum value of $k$ for which $G$ has an acyclic $k$-coloring. Let $a(n)$ denote the maximum value of the acyclic chromatic number among all graphs of genus $n$. In [5], Grünbaum conjectured that $a(0)=5$ and proved that $a(0) \leq 9$. The conjecture was proved by Borodin [3] after the upper bound was improved three times in [7], [1] and [6]. In [2], we proved that $a(1) \leq a(0)+3$. The purpose of this paper is to prove the following:

THEOREM. Any graph of genus $n>0$ can be acyclically colored with $4 n+4$ colors.

It is not known for any $n>0$ whether $a(n)>H(n)$, the Heawood number [8].

2. Preliminaries. The proof of the theorem is by a double induction on $n$, the genus, and $V$, the number of vertices. Since the theorem is true for $n=1$ [2] and trivally true if $V \leq 4 n+4$, the induction begins. Let $G$ be a graph with $V$ vertices which is 2-cell imbedded on $S_{n}$, the $n$-handle sphere, (i.e. every region is homeomorphic to a disc). The inductive hypotheses will be that any such graph of genus at most $n-1$ can be acyclically colored with $4 n$ colors and that any graph of genus $n$ with fewer than $V$ vertices can be acyclically colored with $4 n+4$ colors. The induction will proceed using the concept of reducibility.

A graph $H$ is said to be acyclically $k$-color reducible if, whenever a graph $J$ contains $H$ as a subgraph, we can define a graph $J^{\prime}$ having fewer vertices than $J$ and having the property that given any acyclic $k$-coloring of $J^{\prime}$ we can obtain an acyclic $k$-coloring of $J$. A graph $H$ is said to be reducible if it is acyclically $k$-color reducible for every $k \geq 7$. Clearly, showing that $G$ contains a reducible graph would suffice to prove the theorem. We restate a result proved in [1].

Lemma 1. Let $C$ be a 4-cycle enclosing a planar region. Let $L$ be the set of vertices interior to $C$. If $H$, the induced subgraph on the vertices of $L \cup C$, is a triangulation of the interior of $C$ and if $L$ has more than one vertex, then $H$ contains a reducible graph.

We call a cycle $C \subset G$ contractible (resp. non-contractible) if it is (resp. is not) homotopic to a point.

Lemma 2. Let $C=c_{1}, c_{2}, \ldots, c_{r}$ be a non-contractible cycle in $G$ that does not separate $S_{n}$. Then $G-C$ has genus at most $n-1$. Grant.

* Research supported in part by the Research Corporation through a Cottrell College Science Research Glasgow Math. J. 19 (1978) 163-166. 
Proof. Define graph $G^{*}$ by slitting $C$ into two independent parallel cycles $C^{\prime}=$ $c_{1}^{\prime}, \ldots, c_{r}^{\prime}$ and $C^{*}=c_{1}^{*}, \ldots, c_{r}^{*}$ leaving all incident edges intact.

Now define graph $G^{\prime}$ by taking $G^{*}$ and "cutting" the surface of $S_{n}$ along a simple closed curve through the strip between $C^{\prime}$ and $C^{*}$. Then patch the surface by "pasting" discs into $C^{\prime}$ and $C^{*}$, creating two new faces bounded by $C^{\prime}$ and $C^{*}$, respectively. $G^{\prime}$ is 2-cell imbedded on the new surface.

If $G$ has $V$ vertices, $E$ edges and $F$ faces, then $G^{\prime}$ has $V+r$ vertices, $E+r$ edges and $F+2$ faces. Let $n^{\prime}$ be the genus of $G^{\prime}$. By Euler's formula,

$$
\begin{aligned}
2-2 n^{\prime} & =(V+r)-(E+r)+(F+2) \\
& =V-E+F+2 \\
& =2-2 n+2 .
\end{aligned}
$$

Thus, $n^{\prime}=n-1$. Since $G^{\prime}$ has genus $n-1, G-C=G^{\prime}-\left(C^{\prime} \cup C^{*}\right)$ has genus at most $n-1$.

Lemma 3. Let $C$ be a non-contractible (simple) cycle in $G$ that separates $S_{n}$. Then $G-C$ is the union of two graphs, each of genus at most $n-1$.

Proof. When we perform the same "cut and paste" operation used in the proof of Lemma 2 the resulting graph $G^{\prime}$ is disconnected. Say $G^{\prime}=G_{1} \cup G_{2}$, where $G_{1}$ and $G_{2}$ are 2-cell imbedded on their respective surfaces with $G_{1}$ having $V_{1}$ vertices, $E_{1}$ edges, $F_{1}$ faces, and genus $n_{1} ; G_{2}$ having $V_{2}$ vertices, $E_{2}$ edges, $F_{2}$ faces, and genus $n_{2}$.

As in the proof of Lemma 2,

$$
V_{1}+V_{2}=V+r, \quad E_{1}+E_{2}=E+r \text { and } F_{1}+F_{2}=F+2 .
$$

Then

$$
\begin{aligned}
2-2 n & =V-E+F \\
& =(V+r)-(E+r)+F \\
& =\left(V_{1}+V_{2}\right)-\left(E_{1}+E_{2}\right)+\left(F_{1}+F_{2}-2\right) \\
& =\left(V_{1}-E_{1}+F_{1}\right)+\left(V_{2}-E_{2}+F_{2}\right)-2 \\
& =\left(2-2 n_{1}\right)+\left(2-2 n_{2}\right)-2 .
\end{aligned}
$$

So $n_{1}+n_{2}=n$. Since $C$ is non-contractible neither $n_{1}$ nor $n_{2}$ can be zero. Thus $G^{\prime}$ and, hence, $G-C=G^{\prime}-\left(C^{\prime} \cup C^{*}\right)$ is the union of two graphs each of genus at most $n-1$.

3. Proof of the Theorem. Let $C=c_{1}, \ldots, c_{r}$ be a non-contractible cycle of minimum length in $G$. Depending on whether $C$ does or does not separate $S_{n}$, we apply Lemma 2 or Lemma 3 to show that $G-C$ either is the disjoint union of two graphs of genus at most $n-1$, or else is itself of genus at most $n-1$. In either case we can apply the inductive hypothesis to show that $G-C$ can be acyclically colored with $4 n$ colors.

We show that if $G$ contains no reducible subgraph then the vertices of $C$ can be replaced and colored with four new colors in such a fashion that $G$ will contain no two-colored cycle. This is done in three cases depending on $r$, the length of $C$. 
(i) If $r \leq 4$, use a new color for each point of $C$. No two-colored cycle can be introduced as each of the new colors occurs only once in $G$.

For the next case we assume $G$ is a triangulation. If $G$ is not a triangulation the addition of edges to $G$ cannot decrease $a(G)$. If edges cannot be added to $G$ to make it a triangulation then $G$ contains a pair of vertices, say $x$ and $y$, such that $G-x-y$ has genus less than $n$. The proof would then proceed as in (i), using instead of $C$ the subgraph $\{x, y\}$.

(ii) Assume $G$ is a triangulation and $r=5$. Color $c_{1}, \ldots, c_{5}$ with four new colors $a, b, a, c, d$ respectively. The only two-color cycle that can be introduced is a four-cycle of the form $C^{\prime}=c_{1}, p, c_{3}, q . C^{\prime}$ must be contractible as we assumed $C$ was a minimum length non-contractible cycle. Thus, there can be at most one vertex interior to $C^{\prime}$. Otherwise Lemma 1 guarantees that $G$ contains a reducible graph.

If $C^{\prime}$ has no interior vertices, then since $G$ is a triangulation, either $c_{1}$ is adjacent to $c_{3}$ or $p$ is adjacent to $q$. But in the first instance $C$ was not the shortest non-contractible cycle and in the second instance the acyclic coloring of $G^{\prime}$ was not proper. Thus we may assume there is exactly one point, say $x$, inside $C^{\prime}$.

By the minimality of $C$, both $c_{1}, c_{2}, c_{3}, p$ and $c_{1}, c_{2}, c_{3}, q$ must be contractible. But then either $c_{1}, c_{2}, c_{3}, p$ contains $x$ and $q$ in its interior, or else $c_{1}, c_{2}, c_{3}, q$ contains $x$ and $p$ in its interior. We invoke Lemma 1 to show that $G$ contains a reducible graph.

(iii) If $r \geq 6$, color the vertices of $C$ according to the following prescription. Construct the graph $C^{2}$ whose vertices are the vertices of $C$, with edges joining two vertices of $C^{2}$ if the vertices are of distance one or two in the graph induced on the vertices of $C$. Since the latter graph is regular of degree two ( $C$ can have no diagonals), $C^{2}$ is regular of degree four. Since $C^{2}$ has maximum degree four and does not contain $K_{5}$, Brooks' Theorem [4] implies that $C^{2}$ can be properly four-colored.

When replacing $C$ into $G$, color it with four new colors according to the proper four-coloring of $C^{2}$. The only two-color cycle which can be introduced is of the form $\ldots c_{i}, p, c_{j}, \ldots$, where we assume $i<j$.

Since $c_{i}$ and $c_{j}$ are colored the same they cannot be adjacent in $C^{2}$; thus they must have at least two vertices between them along $C$. Now consider the cycles $c_{1}, \ldots, c_{i}, p, c_{j}, \ldots, c_{r}$ and $c_{i}, c_{i+1}, \ldots, c_{j}, p$. Both have length less than $r$, and at least one is non-contractible since their mod 2 sum is $C$. Thus no two-color cycles can be introduced and the theorem is proved.

\section{REFERENCES}

1. M. O. Albertson and D. M. Berman, Every planar graph has an acyclic 7-coloring, Israel J. Math., 28 (1977), 169-174.

2. M. O. Albertson and D. M. Berman, The acyclic chromatic number, Proc. 7th S-E Conf. Combinatorics, Graph Theory, and Computing (Utilitas Math., 1976), 51-60.

3. O. V. Borodin, A proof of $\mathbf{B}$. Grünbaum's conjecture on the acyclic 5-colorability of planar graphs (Russian), Dokl. Akad. Nauk SSSR 231 (1976), 18-20.

4. R. L. Brooks, On coloring the nodes of a network, Proc. Cambridge Philos. Soc. 37 (1941), 194-197.

5. B. Grünbaum, Acyclic colorings of planar graphs, Israel J. Math. 14 (1973), 390-408. 
6. A. V. Kostochka, Acyclic 6-coloring of planar graphs (Russian), Diskret. Analiz. 28 (1976), 40.

7. J. Mitchem, Every planar graph has an acyclic 8-coloring, Duke Math. J. 41 (1974), 177-181.

8. G. Ringel, Map Color Theorem (Springer, 1974).

Department of Mathematics

SMith COLlege

NoRTHAMPTON

MASs. 01063
Department of Mathematics UNIVERSITY OF NEW ORLEANS NeW ORLEans

LA. 70122 